\title{
Propofol induces apoptosis of hepatocellular carcinoma cells by upregulating miR-134 expression
}

\author{
Xueyan $\mathrm{Hu}^{1}$, Xiaodong $\mathrm{Hu}^{2}$, Qinghui Wang ${ }^{3}$ \\ ${ }^{1}$ Department of Anesthesiology, Linyi Central Hospital, Linyi, China; ${ }^{2}$ Department of Anesthesiology, Linshu County People's Hospital, Linyi, \\ China; ${ }^{3}$ Department of Anesthesia and Perioperative Medicine, Maternity and Child Health Care of Zaozhuang, Zaozhuang, China \\ Contributions: (I) Conception and design: All authors; (II) Administrative support: All authors; (III) Provision of study materials or patients: All \\ authors; (IV) Collection and assembly of data: All authors; (V) Data analysis and interpretation: All authors; (VI) Manuscript writing: All authors; (VII) \\ Final approval of manuscript: All authors. \\ Correspondence to: Qinghui Wang. Department of Anesthesia and Perioperative Medicine, Maternity, and Child Health Care of Zaozhuang, No. 25 \\ Wenhua East Road, Shizhong District, Zaozhuang 277100, China. Email: wangweilin-007@163.com.
}

Background: Propofol is an anesthetic used in clinical surgery. Many studies have shown that propofol has the potential to kill cancer cells; however, the mechanism by which it kills hepatocellular carcinoma (HCC) cells remains unclear.

Methods: To examine this issue, the apoptosis change of HepG2 and Huh-7 cell lines treated with propofol were observed. Additionally, a quantitative reverse-transcriptase polymerase chain reaction was used to measure the expression level of hsa-miR-134 (miR-134), and a Cell Counting Kit-8 assay and flow cytometry assay were used to observe cell apoptosis. A dual-luciferase assay was used to confirm the binding effect of miR-134 and BCL-2 (B cell lymphoma-2), and a western blot assay was used to detect expression level changes of BCL-2 and cleaved caspase-3.

Results: The results showed that propofol significantly promoted HepG2 and Huh-7 cell apoptosis, and that miR-134 expression level is related to the concentration of propofol. The dual-luciferase assay showed that miR-134 significantly reduced the luciferase activity of BCL-2-wt, but had no notable effect on BCL2-mut. In rescue experiments, miR-134 deficiency resulted in a high apoptosis rate, a low BCL-2 expression level, and a high cleaved caspase-3 expression level induced by propofol in HepG2.

Conclusions: In summary, propofol appears to upregulate the expression level of miR-134, decrease the BCL-2 level, and induce HCC cell apoptosis by promoting the cleaved caspase-3 expression level.

Trial Registration: Chinese Clinical Trial Registry ChiCTR18000199

Keywords: Propofol; mir-134; hepatocellular carcinoma (HCC); apoptosis; BCL-2

Submitted Apr 16, 2021. Accepted for publication Jun 15, 2021.

doi: $10.21037 /$ tcr-21-830

View this article at: https://dx.doi.org/10.21037/tcr-21-830

\section{Introduction}

Hepatocellular carcinoma (HCC) is one of the most common cancers in the world and has high morbidity and mortality. Indeed, HCC is a common malignant disease, and its incidence increases year by year $(1,2)$. Developing countries have a high incidence of liver diseases. Factors contributing to liver cancer include the infections induced by hepatitis $\mathrm{B}$ and hepatitis $\mathrm{C}$ virus infections, the problems caused by bad habits, fatty liver disease induced by various dietary exposures and alcohol-related cirrhosis (3-5). In clinical terms, surgery, radiotherapy, and chemotherapy are used to treat liver cancer patients; however, their effects are limited in most cases (6). Research has indicated that surgical resection is only suitable for a few early-stage patients with liver cancer, and right hepatectomy has a higher risk of post-operative complications than left hepatectomy (7). Drug chemotherapy, such as sorafenib which is the most common option for late- 
stage patients, could delay the deterioration of liver cancer in some advanced patients, but the resistance of the tumor cells on drugs causes poor prognosis of the patients (8). As a result, conventional therapy methods have limited effects in improving the outcomes of this dangerous disease. Further research needs to be conducted to find better methods for treating liver cancer.

Propofol (2,6-diisopropylphenol) is currently one of the most commonly used anesthetics in clinical practice. It was first introduced into clinical treatment in 1986, and served as an anesthetic for surgery (9). Notably, in recent years, propofol has not only served as a sedative or hypnotic drug during surgery, but has also been shown to have many non-anesthetic effects, such as anti-tumor or carcinogenic activities $(10,11)$. Many reports have indicated that the anti-tumor effects of propofol are closely related to micro ribonucleic acids (miRNAs). For example, propofol could downregulate miR-372 to suppress the activity of Wnt/ $\beta$-catenin pathway and mechanistic target of rapamycin (mTOR) signal pathways, and finally inhibit non-small cell lung cancer cell invasion and migration (12). In breast cancer, propofol could downregulate miR-21 and miR-24 to inactivate phosphatidylinositol-3-Kinase and Protein Kinase $\mathrm{Ba}, \mathrm{Wnt} / \beta$-catenin and $\mathrm{p} 27$, and finally inhibit cell proliferation and epithelial-mesenchymal transition $(13,14)$.

In this study, we examined the effects of propofol in inducing HCC cell apoptosis, and illuminated the mechanism by which propofol induces HCC apoptosis progress to provide some reference for HCC research and treatment. We present the following article in accordance with the MDAR reporting checklist (available at https:// dx.doi.org/10.21037/tcr-21-830).

\section{Methods}

\section{Cell culture}

HCC cell lines, including HepG2 and Huh-7, were used in this study (all of which were purchased from $\mathrm{BeNa}$ Culture Collection, Beijing, China). All cells were cultured with Dulbecco's Modified Eagle Medium (HyClone Logan, State of Utah, USA) and 10\% fetal bovine serum (FBS) purchased from PAN Biotech (Shanghai, China) in an incubator $\left(37^{\circ} \mathrm{C}\right.$ and $5 \%$ carbon dixoide).

\section{Cell transfection}

When the density of cells reached $70 \%$ in each well, the miR-134 inhibitor was transfected into wells with lipofectamine 3000 (Invitrogen, California, USA), and the cells were then incubated for $48 \mathrm{~h}$.

\section{Western blot}

The total protein of cells was extracted with a radioimmunoprecipitation assay buffer. The extracts were added with $1 \%$ phenylmethylsulfonyl fluoride (Beyotime, Shanghai, China), and then were used for western blot assay. A Pierce bicinchoninic acid (BCA) protein assay kit (Beyotime, Shanghai, China) was used to measure protein concentration. Target proteins in the samples were separated by $10 \%$ sodium dodecyl sulphate-polyacrylamide gel electrophoresis (SDS-PAGE), and then transferred from SDS-PAGE onto polyvinylidene fluoride or polyvinylidene difluoride membranes. In addition, $\beta$-actin was used for the endogenous controls. After blocking with $5 \%$ nonfat milk for $1 \mathrm{~h}$, the membranes were incubated with the primary antibodies at $4{ }^{\circ} \mathrm{C}$ overnight. After being washed 3 times with tris-buffered saline, the membranes were incubated with secondary antibodies for $1.5 \mathrm{~h}$ at $25^{\circ} \mathrm{C}$. Finally, the chemiluminescence detection system was used to observed protein samples. The antibodies were used as follow: anti-BCL-2 (1:1000, ab10978135, ThermoFisher, Massachusetts, USA); anti-cleaved Caspase-3 (1:1000, ab10979528, ThermoFisher, Massachusetts, USA); anti- $\beta$ actin (1:1000, sc-47,778, Santa Cruz).

\section{$q R T-P C R$}

The total RNA of cells was extracted with a Trizol reagent, and then used for the reverse transcription with the Revert Aid First Strand cDNA Synthesis Kit (Thermo Fisher, Massachusetts, USA). Primers of miR-134 were synthesized and purified by RiboBio (Guangzhou, China). U6 small nuclear RNA (U6) was used as the endogenous control. The information of primers was list in Table 1.

\section{Flow cytometry assay}

The treated human brain microvascular endothelial cells (HBMECs) were treated with trypsinase $(0.25 \%$, ethylenediaminetetraacetic acid-free) and harvested. After being washed 3 times with ice phosphate-buffered saline, a total of $1 \times 10^{5}$ cells were diluted in ice Annexin V-FITC binding buffer. Subsequently, the HBMECs were incubated with Annexin V-FITC and propidium iodide in darkness 
for $15 \mathrm{~min}$ at room temperature. Next, a flow cytometer (BD Biosciences, State of New Jersey, USA) was used to determine the cell apoptosis ratio.

\section{CCK-8}

The HBMECs were seeded into 96-well plates. After transfection, the cells in each well were incubated for $48 \mathrm{~h}$ and the Cell Counting Kit-8 (CCK-8) solution (Amyjet, Wuhan, China) was then added. In addition, a blank well was added to the CCK-8 solution as a control to standardize the value of all the wells. After incubating for $4 \mathrm{~h}$, the absorbance value was measured using a microplate reader (Flash, Shanghai, China) at $450 \mathrm{~nm}$.

\section{Statistical analysis}

The data are listed as mean \pm standard deviation (SD).

Table 1 Primer sequence of miR-134, BCL-2 and U6

\begin{tabular}{lc}
\hline Name of primer & \multicolumn{1}{c}{ Sequences } \\
\hline miR-134-F & 5'-CTGTGGGCCACCTAGTCACCAA-3' \\
miR-134-R & 5'-GCTGTCAACGATACGCTACCTA-3' \\
BCL-2-F & 5'-ACTGGCTCTGTCTGAGTAAG-3' \\
BCL-2-R & 5'-CCTGATGCTCTGGGTAAC-3' \\
U6-F & 5'-CTCGCTTCGGCAGCACA-3' \\
U6-R & 5'-AACGCTTCACGAATTTGCGT-3' \\
\hline
\end{tabular}

A

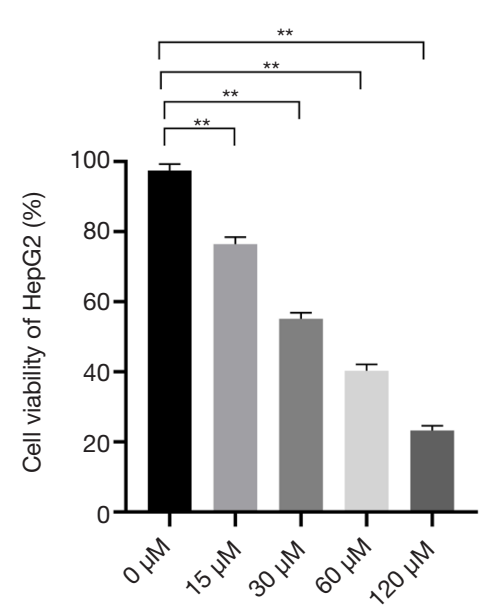

SPSS 13.0 and Graphpad Prism 8.0 were used to analyze and display the results. The differences between the groups were calculated using a Chi-squared test or analysis of variance with Tukey's post hoc-test. All of the experiments were performed at least 3 independent times. A $\mathrm{P}$ value $<0.05$ was considered statistically significant.

\section{Results}

\section{Propofol promotes HepG2 cell apoptosis}

To analyze the lethal effect of propofol on HCC cells, HepG2 and Huh-7 were treated with 5 gradient concentrations of propofol (i.e., 0, 15 30, 60, and $120 \mu \mathrm{M})$, and a CCK-8 assay was used to observe the viability ratio of the cells. As Figure 1 shows, the apoptosis rates of HepG2 and Huh-7 cells were dose-dependent; as propofol concentrations increased, the apoptosis ratio of the cells also increased. According to the results, $30 \mu \mathrm{M}$ was the half-maximal inhibitory concentration of propofol in HepG2 cells, and $60 \mu \mathrm{M}$ was the half-maximal inhibitory concentration of propofol in Huh-7 cells.

\section{Propofol promotes the expression of cleaved casepase- 3 and B cell lymphoma-2 (BCL-2) in HepG2 cells}

To further explore the mechanism by which propofol has a lethal effect on HCC cells, the HepG2 cell line was selected as the experimental subject, and $30 \mu \mathrm{M}$ of propofol was used to treat the HepG2 cells. Additionally, a western blot assay

B

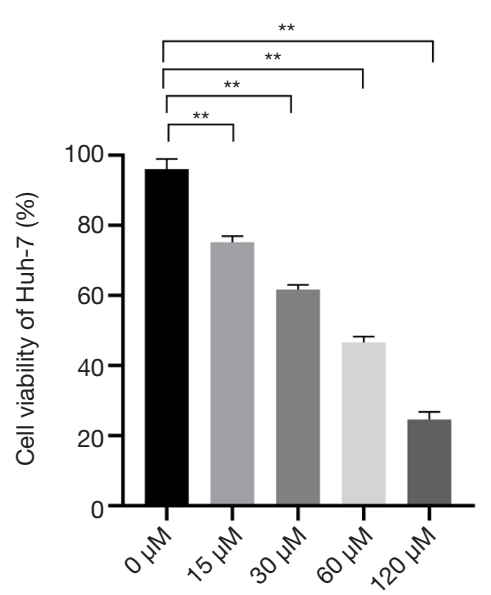

Figure 1 The viability of HepG2 and Huh7 is significantly related to the concentration of propofol. The cell viability ratio was detected using a CCK-8 assay. (A) HepG2; and (B) Huh-7. **, $\mathrm{P}<0.01$. 
A

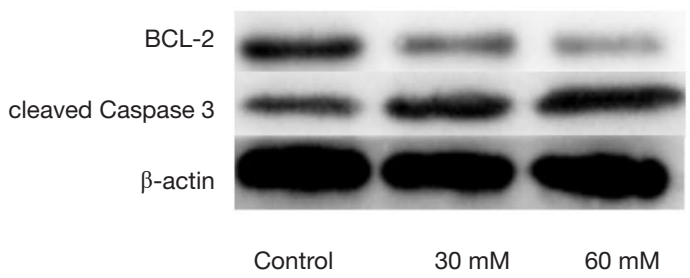

B

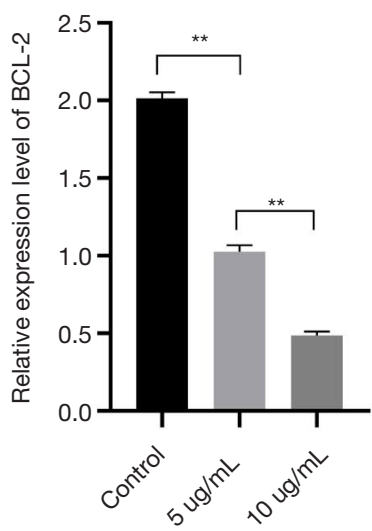

C

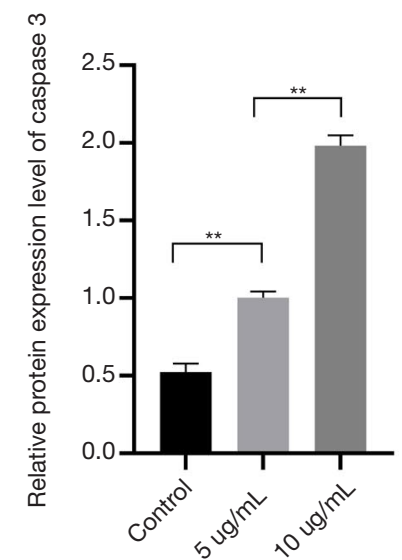

Figure 2 Propofol promotes the expression of cleaved casepase-3 and BCL-2 (B cell lymphoma-2) in HepG2 cells. A western blot assay was used to examine the effects of propofol on the expression of caspase-3 and BCL-2. (A) The expression level of BCL-2 and cleaved caspase-3. (B) The relative expression level of BCL-2. (C) The relative expression level of caspase-3. **, $\mathrm{P}<0.01$.

was used to detect the expression level of caspase-3 and BCL-2. The results showed that compared to the control group, the expression level of BCL-2 was decreased, but the expression level of cleaved caspase- 3 was significantly increased. Thus, propofol appeared to effectively promote HepG2 apoptosis (see Figure 2; $\mathrm{P}<0.05$ ).

\section{Propofol promotes miR-134 expression in HepG2 cells}

To analyze the relationship between propofol and miR134, different concentrations of propofol were used to treat the HCC cells, and a quantitative reverse-transcriptase polymerase chain reaction (qRT-PCR) was used to detect the expression level of miR-134. As Figure $3 A$ shows, after propofol treatment, compared to the control group, the miR-134 expression levels of the HepG2 cells in both the 30 and $60 \mu \mathrm{M}$ treatment groups were significantly increased $(\mathrm{P}<0.05)$. The expression pattern of miR-134 in the Huh7 cells showed similar results (see Figure $2 B ; \mathrm{P}<0.05$ ). Thus, the results indicate that propofol stimulated the expression of miR-134.

\section{MiR-134 directly targets $B C L-2$}

The database of miRNAs target was used to predict the downstream targets of miR-134, and the results showed BCL-2 was a potential target of miR-134. The qRT-PCR showed that miR-134 was significantly upregulated in the cells treated with propofol (see Figure $3 A ; \mathrm{P}<0.05$ ). To further confirm their connection activity, a dual-luciferase assay was used to detect the combination abilities of miR134 to wild and mutation types of BCL-2. The results showed that miR-134 significantly decreased the luciferase activity of BCL-2-wt (see Figure $3 B ; \mathrm{P}<0.05$ ), but had no effect on BCL-2-mut (see Figure $3 B$; $>>0.05$ ). These results suggested that miR-134 directly targets BCL-2.

\section{Downregulated miR-134 reversed the effect of propofol in inducing HepG2 cell apoptosis}

To confirm the role of miR-134 in the process of propofolinducing HCC cell apoptosis, a miR-134 inhibitor was transfected into HepG2 cells, which were treated with 30 $\mu M$ propofol at the same time. In the experiments, CCK8 was used to observe the cell viability ratio, and a western blot assay was used to detect the expression level of BCL-2 and cleaved caspase-3. The results of the CCK- 8 and flow cytometry assay showed that the apoptosis level increased significantly in the group that was treated with propofol only (see Figure $4 A, B, C$; all $\mathrm{P}<0.05$ ). Conversely, the group that was treated with propofol and transfected with a miR134 inhibitor showed only a subtle difference compared to the control group (see Figure $4 A, B, C$; all $\mathrm{P}<0.05$ ). The western blot assay showed that the expression level of BCL- 
A

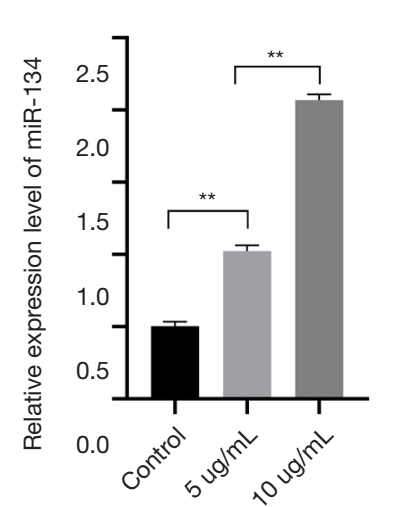

B

miR-134 5'-CCUGUGgGCCACCUAGUCA-3' II II IIII BCL-2 5'-GTCCACCCGGTTCCGGTGTG-3'
C

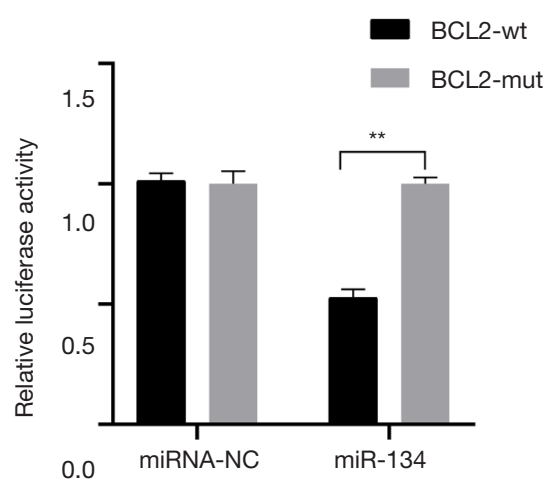

Figure 3 Propofol promotes miR-134 expression in HepG2 cells, and miR-134 directly targets 3'-UTR of BCL-2. A QRT-PCR was used to detect the expression level of miR-134, and a dual-luciferase assay was used to observe the binding effect of miR-134 and BCL-2 (B cell lymphoma-2). (A) The relative expression level of miR-134. (B) Binding sites of miR-134 and BCL-2 (B cell lymphoma-2). (C)The relative luciferase activities of BCL-2-wt and BCL-2-mut. **, $\mathrm{P}<0.01$.

A

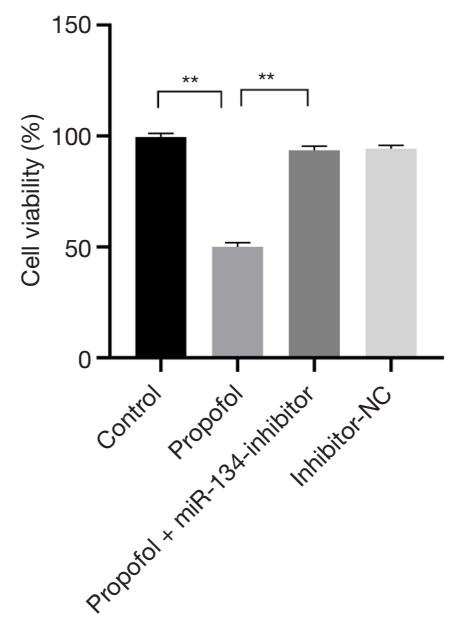

B
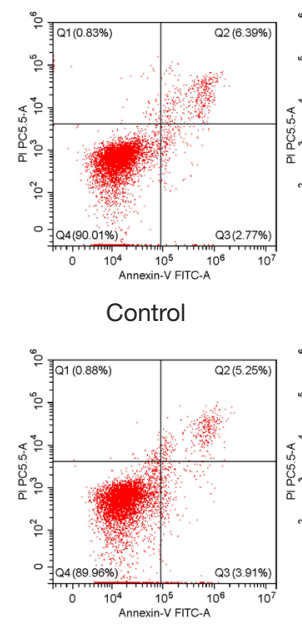

Propofol + miR-134-inhibitor
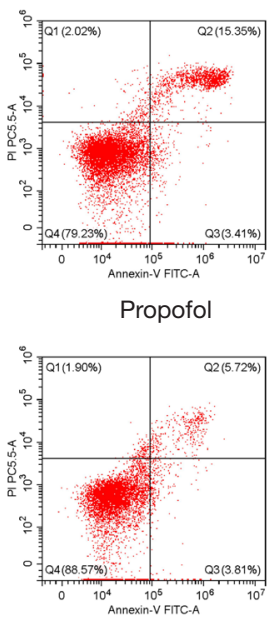

Inhibitor-NC
C

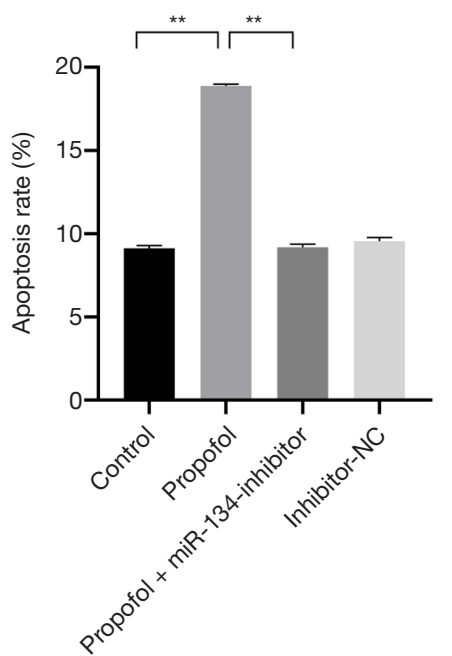

Figure 4 Downregulated miR-134 reversed the effect of propofol in inducing HepG2 cell apoptosis. The CCK-8 and flow cytometry assay were used to observe the viability and apoptosis of cells. (A) Cell viability. (B-C) Flow cytometry assay. ${ }^{* *}, \mathrm{P}<0.01$.

2 decreased and the expression level of cleaved caspase-3 increased in the group that was treated with propofol only (see Figure 5; $\mathrm{P}<0.05$ ). Conversely, the expression level had no obvious change in the group that was treated with propofol and transfected with miR-134; however, the inhibitor group showed a subtle difference compared to the control group (see Figure 5; $\mathrm{P}<0.05$ ). The results suggest that a miR-134 deficiency reversed the effect of propofol on BCL-2 and cleaved caspase-3 to induce HepG2 cell apoptosis.

\section{Discussion}

The mechanism of HCC formation and development 
A

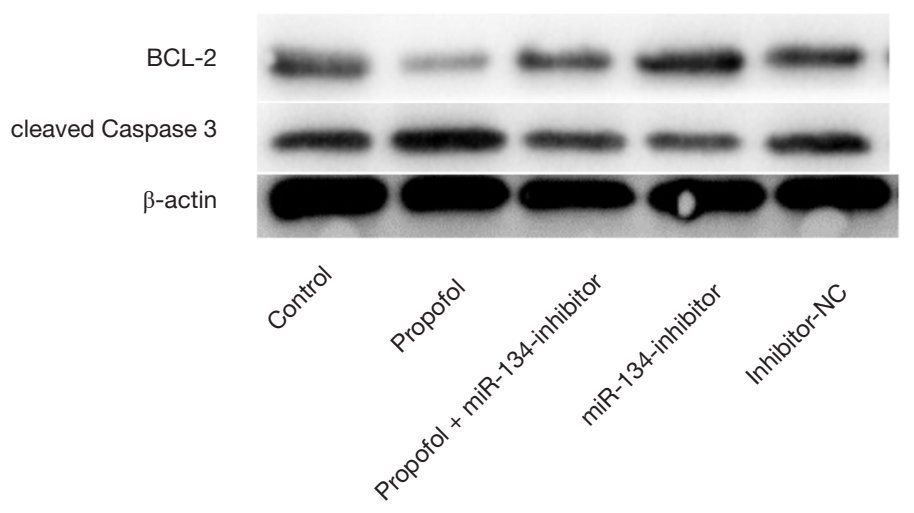

B

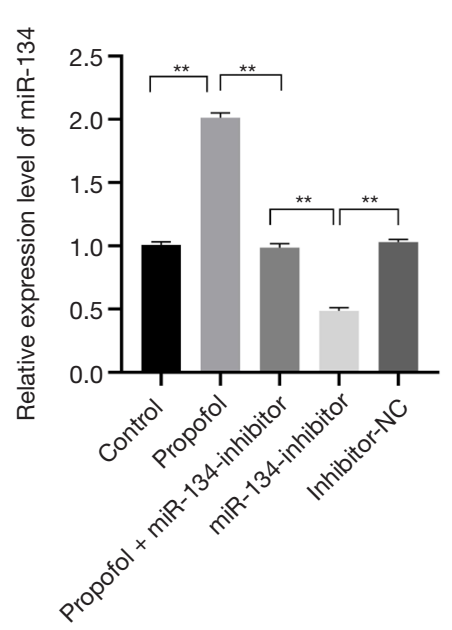

C

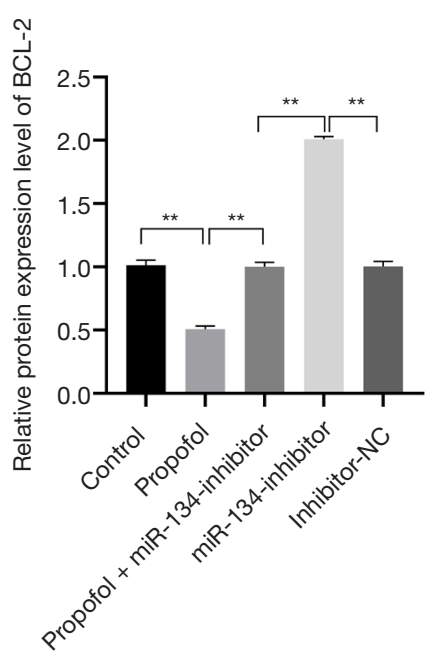

D

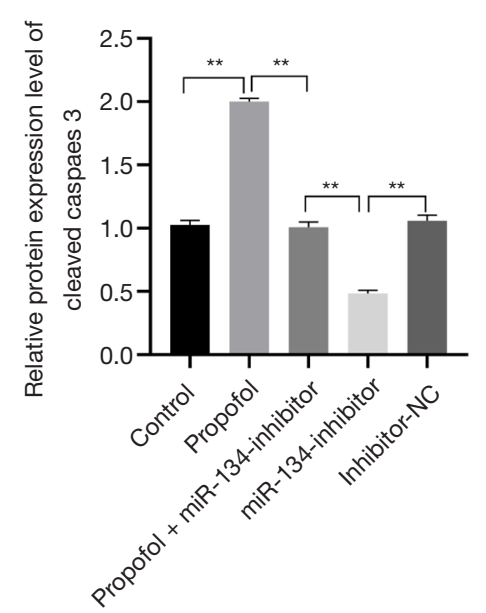

Figure 5 Downregulated miR-134 reversed the effect of propofol on downregulating BCL-2 (B cell lymphoma-2) and upregulating cleaved caspase-3. A western blot assay was used to examine the effect of miR-134 deficiency on the expression of caspase-3 and BCL-2. (A) The expression level of BCL-2 and cleaved caspase-3. (B) The expression level of miR-134. (C) The relative expression level of BCL-2. (D) The relative expression level of caspase- $3 .{ }^{* *}, \mathrm{P}<0.01$.

is complex. It is related to the regulation of multiple pathways. As the current clinical routine treatment has some limitations, a new and effective treatment is urgently necessary. Anesthesia is widely used in surgery to effectively reduce the pain of patients. In recent research, some current clinical anesthetics have been found to have anti-cancer effects $(13,15)$. Propofol is a safe and an effective alternative for sedation. Propofol has been reported to have some advantages, including a rapid onset and a short duration of action. Thus, the use of intravenous anesthesia with propofol is growing in popularity. However, propofol plays an effective role in tumor inhibition, which could hinder liver cancer cell invasion, migration, and proliferation, and induce cell apoptosis. For example, Du et al. found that propofol targets Transcription factor SOX-4 (SOX4) to significantly decrease endometrial cancer cell colony numbers, inhibit their viability, migration, and invasion, and promote cancer cell apoptosis in a dose-dependent manner (16). To explore the lethal effect of propofol on liver cancer cells, we treated HCC cells with different concentrations of propofol, and found that propofol promoted HCC cell apoptosis in a dose-dependent manner.

Several studies have shown that propofol might regulate the biological behaviors of cancer cells by upregulating 
or downregulating the expression of some miRNAs. Li et al. (17) found that propofol inhibited colorectal cancer cell invasion and migration, and induced cell apoptosis by regulating the miR-124-3p/AKT pathway. Yu et al. (18) suggested that propofol could effectively inhibit pancreatic cancer proliferation and metastatic abilities by upregulating miRNA-328 and downregulating disintegrin and metalloproteinase domain-containing protein 8 (ADAM8) expression. Sun et al. (12) found that propofol could inhibit the proliferation and invasion of A549 cells by regulating miR-372 expression. Additionally, propofol could be combined with other drugs to enhance their treatment effects on cancer cells. For example, Zhang et al. found that propofol could inhibit gastric cancer cell autophagy to weaken the resistance of cancer cells to cisplatin by regulating the lncRNA MALAT1/miR-30e/ATG5 axis (19).

MiRNAs are small endogenous non-coding RNAs, which have been proven to be related to the proliferation, invasion, and migration of many cancers (20-22). MiRNAs are involved in many life activities of cells, and research has indicated that there is an immediate connection between miRNAs abnormal expression and cancer cell invasion and proliferation. Additionally, the functions of different miRNAs are diverse. Studies have found that overexpressed miR-26a-5p could inhibit gastric cancer cell proliferation and invasion abilities by targeting Wnt5a, while miR-20a could inhibit colorectal cancer cell autophagy by targeting ATG5/FIP200 $(23,24)$. In the present study, we found that propofol promoted miR-134 expression in HCC cells, and the miR-134 expression level is related to the proliferation abilities of HCC cells, which further suggests that propofol affects cancer cells' life activities by regulating the expression of miRNAs to some degree. To further analyze the regulation relationship between propofol and miR134 , we conducted reserve experiments to confirm whether miR-134 was necessary for propofol to induce HCC cell apoptosis procession. We found that the suppression of miR-134 by transfection with a miR-134 inhibitor reversed the effects of propofol on liver cancer cell apoptosis. These results indicate that the anti-tumor function of propofol might be partly related to miR-134 upregulation.

Multiple studies have indicated that miR-134 acts as a tumor inhibitor in many cancers. The downregulation of miR-134 might induce cancer cell proliferation and invasion by targeting the mRNAs of several proteins. For example, one study indicated that miR-134 targeted 3'UTRs of matrix metalloproteinase 1 (MMP1) and matrix metalloproteinase 3 (MMP3), and further inhibited osteosarcoma cell invasion and metastasis (25). Additionally, miR-134 was found to have a connection to liver cancer. He et al. (26) found that miR-134 targets FOXM1 to inhibit HCC cell proliferation. In this study, we observed that propofol promoted caspase-3 activity, while miR134 deficiency induced by transfecting miR-134 inhibitor reserved this situation. Caspase- 3 is a key protein in cell apoptosis, which plays an important role in the processes of tissue development and differentiation $(27,28)$. In addition, we found that miR-134 directly targets BCL-2 and reduces its expression level in HCC cells. BCL-2 is an apoptosisrelated protein that inhibits cytochrome $\mathrm{C}$ release by its downstream factor, BCL-2 associated X (Bax), to further reduce the activity of caspase-3, and finally inhibit cell apoptosis (29). Based on these findings, propofol appears to upregulate miR-134 expression, inhibit BCL-2 expression level, and finally promote HCC cell apoptosis.

We explained the mechanism by which propofol has a lethal effect on HCC cells; however, the deep relationship between propofol and miR-134 remains unknown, and we are still not sure how propofol upregulates miR-134 expression levels in cancer cells. Thus, further research is needed. Additionally, for a more credible conclusion, it is necessary that more evidence about the propofol and miR-134 connection be gathered by in vivo animal model experiments.

\section{Conclusions}

In summary, propofol appears to upregulate the miR134 expression level, inhibit BCL-2 expression, promote caspase- 3 activation, and induce HCC cell apoptosis.

\section{Acknowledgments}

Funding: None.

\section{Footnote}

Reporting Checklist: The authors have completed the MDAR reporting checklist. Available at https://dx.doi. org/10.21037/tcr-21-830

Data Sharing Statement: Available at https://dx.doi. org/10.21037/tcr-21-830

Conflicts of Interest: All authors have completed the ICMJE uniform disclosure form (available at https://dx.doi. org/10.21037/tcr-21-830). The authors have no conflicts of 
interest to declare.

Ethical Statement: The authors are accountable for all aspects of the work in ensuring that questions related to the accuracy or integrity of any part of the work are appropriately investigated and resolved. The study was approved by Linyi Central Hospital (No. LY20200115).

Open Access Statement: This is an Open Access article distributed in accordance with the Creative Commons Attribution-NonCommercial-NoDerivs 4.0 International License (CC BY-NC-ND 4.0), which permits the noncommercial replication and distribution of the article with the strict proviso that no changes or edits are made and the original work is properly cited (including links to both the formal publication through the relevant DOI and the license). See: https://creativecommons.org/licenses/by-nc-nd/4.0/.

\section{References}

1. Bray F, Ferlay J, Soerjomataram I, et al. Global cancer statistics 2018: GLOBOCAN estimates of incidence and mortality worldwide for 36 cancers in 185 countries. CA Cancer J Clin 2018;68:394-424.

2. Nakagawa H, Fujita M, Fujimoto A. Genome sequencing analysis of liver cancer for precision medicine. Semin Cancer Biol 2019;55:120-7.

3. Tu T, Bühler S, Bartenschlager R. Chronic viral hepatitis and its association with liver cancer. Biol Chem 2017;398:817-37.

4. Arem H, Loftfield E, Saint-Maurice PF, et al. Physical activity across the lifespan and liver cancer incidence in the NIH-AARP Diet and Health Study cohort. Cancer Med 2018;7:1450-7.

5. Szabo G, Saha B, Bukong TN. Alcohol and HCV: implications for liver cancer. Adv Exp Med Biol 2015;815:197-216.

6. Anwanwan D, Singh SK, Singh S, et al. Challenges in liver cancer and possible treatment approaches. Biochim Biophys Acta Rev Cancer 2020;1873:188314.

7. Orcutt ST, Anaya DA. Liver Resection and Surgical Strategies for Management of Primary Liver Cancer. Cancer Control 2018;25:1073274817744621.

8. Yan D, Yan X, Dai X, et al. Activation of AKT/AP1/ FoxM1 signaling confers sorafenib resistance to liver cancer cells. Oncol Rep 2019;42:785-96.

9. Sahinovic MM, Struys MMRF, Absalom AR. Clinical Pharmacokinetics and Pharmacodynamics of Propofol.
Clin Pharmacokinet 2018;57:1539-58.

10. Meng C, Song L, Wang J, et al. Propofol induces proliferation partially via downregulation of $\mathrm{p} 53$ protein and promotes migration via activation of the $\mathrm{Nrf} 2$ pathway in human breast cancer cell line MDA-MB-231. Oncol Rep 2017;37:841-8.

11. Xu W, Zheng J, Bie S, et al. Propofol inhibits Wnt signaling and exerts anticancer activity in glioma cells. Oncol Lett 2018;16:402-8.

12. Sun H, Gao D. Propofol suppresses growth, migration and invasion of A549 cells by down-regulation of miR-372. BMC Cancer 2018;18:1252.

13. Yu B, Gao W, Zhou H, et al. Propofol induces apoptosis of breast cancer cells by downregulation of miR-24 signal pathway. Cancer Biomark 2018;21:513-9.

14. Du Q, Zhang X, Zhang X, et al. Propofol inhibits proliferation and epithelial-mesenchymal transition of MCF-7 cells by suppressing miR-21 expression. Artif Cells Nanomed Biotechnol 2019;47:1265-71.

15. Ye L, Zhang Y, Chen YJ, et al. Anti-tumor effects of lidocaine on human gastric cancer cells in vitro. Bratisl Lek Listy 2019;120:212-7.

16. Du Q, Liu J, Zhang X, et al. Propofol inhibits proliferation, migration, and invasion but promotes apoptosis by regulation of Sox 4 in endometrial cancer cells. Braz J Med Biol Res 2018;51:e6803.

17. Li Y, Dong W, Yang H, et al. Propofol suppresses proliferation and metastasis of colorectal cancer cells by regulating miR-124-3p.1/AKT3. Biotechnol Lett 2020;42:493-504.

18. Yu X, Gao Y, Zhang F. Propofol inhibits pancreatic cancer proliferation and metastasis by up-regulating miR-328 and down-regulating ADAM8. Basic Clin Pharmacol Toxicol 2019;125:271-8.

19. Zhang YF, Li CS, Zhou Y, et al. Propofol facilitates cisplatin sensitivity via lncRNA MALAT1/miR-30e/ATG5 axis through suppressing autophagy in gastric cancer. Life Sci 2020;244:117280.

20. Feliciano A, Garcia-Mayea Y, Jubierre L, et al. miR-99a reveals two novel oncogenic proteins $\mathrm{E} 2 \mathrm{~F} 2$ and EMR2 and represses stemness in lung cancer. Cell Death Dis 2017;8:e3141.

21. Wu Y, Hu G, Wu R, et al. High expression of miR-135b predicts malignant transformation and poor prognosis of gastric cancer. Life Sci 2020;257:118133.

22. Wang W, Yuan X, Xu A, et al. Human cancer cells suppress behaviors of endothelial progenitor cells through miR-21 targeting IL6R. Microvasc Res 2018;120:21-8. 
23. Li Y, Wang P, Wu LL, et al. miR-26a-5p Inhibit Gastric Cancer Cell Proliferation and Invasion Through Mediated Wnt5a. Onco Targets Ther 2020;13:2537-50.

24. Che J, Wang W, Huang Y, et al. miR-20a inhibits hypoxiainduced autophagy by targeting ATG5/FIP200 in colorectal cancer. Mol Carcinog 2019;58:1234-47.

25. Chen CL, Zhang L, Jiao YR, et al. miR-134 inhibits osteosarcoma cell invasion and metastasis through targeting MMP1 and MMP3 in vitro and in vivo. FEBS Lett 2019;593:1089-101.

26. He J, Yang T, He W, et al. Liver X receptor inhibits the growth of hepatocellular carcinoma cells via regulating HULC/miR-134-5p/FOXM1 axis. Cell Signal 2020;74:109720.

Cite this article as: $\mathrm{Hu} \mathrm{X,} \mathrm{Hu} \mathrm{X,} \mathrm{Wang} \mathrm{Q.} \mathrm{Propofol} \mathrm{induces}$ apoptosis of hepatocellular carcinoma cells by upregulating miR-134 expression. Transl Cancer Res 2021;10(6):3004-3012. doi: $10.21037 /$ tcr-21-830
27. Rebellato P, Kaczynska D, Kanatani S, et al. The T-type Ca2+ Channel Cav3.2 Regulates Differentiation of Neural Progenitor Cells during Cortical Development via Caspase-3. Neuroscience 2019;402:78-89.

28. Juraver-Geslin HA, Durand BC. Early development of the neural plate: new roles for apoptosis and for one of its main effectors caspase-3. Genesis 2015;53:203-24.

29. Wu R, Tang S, Wang M, et al. MicroRNA-497 Induces Apoptosis and Suppresses Proliferation via the Bcl-2/BaxCaspase9-Caspase3 Pathway and Cyclin D2 Protein in HUVECs. PLoS One 2016;11:e0167052.

(English Language Editor: L. Huleatt) 